\title{
Transient CFD simulations of turbulent liquid - liquid flow in a Kenics static mixer. Radial and tangential velocities
}

\author{
Halina Murasiewicz, Zdzislaw Jaworski \\ West Pomeranian University of Technology, Institute of Chemical Engineering and Environmental Protection Processes, \\ al. Piastow 42, 71-065 Szczecin, Poland \\ corresponding author: e-mail: Zdzislaw.Jaworski@zut.edu.pl
}

\begin{abstract}
The results of modelling of the two-phase turbulent flow of a two-phase, liquid-liquid mixture in a Kenics static mixer were reported. Advanced transient simulations were performed using the large eddy simulation (LES) approach and a broader analysis of the velocity field was carried out. The two-phase flow was modelled employing the Eulerian approach in the pseudo-homogeneous version of the mixture model. Three cases were again considered, which differed by the density of the two phases and the simulations were performed for Reynolds number of 10,000. The LES results for the tangential and radial components were compared with those obtained in the steady-state RANS approach.
\end{abstract}

Keywords: LES, liquid-liquid, turbulent flow, Kenics mixer.

\section{INTRODUCTION}

Static mixers are widely used for enhancing mixing processes, which are employed in several branches of industry ${ }^{1}$. Industrial mixing is mainly applied for manufacturing stable emulsions, intensifying heat and mass transfer, dispersing insoluble materials, carrying out biochemical reactions and contacting other multiphase systems. Such widespread interest in static mixers results from their low investment and operational costs, small size and more effective local homogenizing than in dynamic (mechanical) mixers.

This paper extends the earlier numerical studies ${ }^{2,3}$ by performing an analysis of the radial and tangential components of the two-phase flow in a Kenics static mixer. In the previous paper $^{3}$ the axial velocity field obtained by means of both numerical methods was presented and discussed. It was found in the study that the area of the fifth mixer insert fully characterizes the developed turbulent flow. Computational Fluid Dynamics (CFD) modelling was used there to predict the turbulent flow conditions of two immiscible liquids. In particular, the local volumetric ratio (concentration) of the liquids was investigated in detail $^{2,3}$.

The homogenisation results of large eddy simulations, LES, were compared in the studies with those for the Reynolds averaging approach, RANS. Both in RANS and LES a significantly lower segregation of the phases was obtained in the case of equal density of the two liquids (case B) than for different phase density - cases A and C. A comparison of the computed relative degree of mixing of the liquids for the A case in RANS and LES indicated a higher phase segregation simulated in RANS. This was attributed to a higher level of the kinetic energy of turbulence predicted in the RANS approach.

Several studies, e.g. ${ }^{4-8}$, were reported in the open literature for the Kenics static mixers using both the experimental and numerical tools. However, no experimental data is available on the local phase concentration in turbulent liquid-liquid (L-L) flows inside static mixers. For the modelling of turbulent flows the numerical methods described thoroughly by Pope ${ }^{9}$ are in use and most often the Reynolds averaged Navier-Stokes (RANS) method is employed. However, the Reynolds time averaging of the transport equations introduces also the closure problem requiring additional models for the fluctuating quantities, like velocity, concentration and temperature. Therefore, knowledge of turbulent velocity fluctuation and energy dissipation, both in the micro and macro scale, becomes of special importance for two - phase flows.

Large eddy simulations and direct numerical simulations (DNS) are now becoming very widely applied in turbulence research due to good accuracy of the description of the main flow characteristics. Direct simulations of turbulence are simply solutions of the time-resolved NavierStokes equations. Nevertheless these are still very much the domain of the specialist research laboratory because they require a huge amount of memory and computing time $^{10}$. The large eddy simulations, LES serve as a reasonable alternative between the DNS and RANS methods. The fundamental assumption here is division of the analyzed vortex scales into two ranges. Eddies larger than numerical cells are resolved directly, whereas the sub-grid scale eddies are usually modelled with the help of the socalled subgrid scale viscosity.

An example of joint application of the LES and RANS analysis to a dynamic mixer can be found in Jahoda et al. ${ }^{11}$. The authors concluded that the LES approach resulted in a better description of the turbulent, single phase flow in the mixer and also significantly better predicted the mixing time necessary for reaching a desired homogenization degree of the stirred mixture. Studies on other mixers were reported by Bakker et al. ${ }^{12}$, who used LES to predict large-scale chaotic structures in the stirred tanks. Their results favourably compared with literature data for single phase flows. Hartmann et al. ${ }^{13}$ carried out flow simulations in stirred tanks also with the help of the RANS and LES methods and confronted the results with the experiments. Again they found that LES delivers a more realistic picture of the flow field and better values of the fluctuation kinetic energy than those from RANS.

Another example of LES applications to static mixers was reported by van Wageningen et al. ${ }^{14}$. They applied the lattice-Boltzmann method to a Kenics mixer and obtained 
a good agreement of the numerical results with the LDA data.

The available research reports on the two-phase flow in static mixers, where the experimental and CFD modelling data are compared, were carried out for the mechanism of drop breakage in the laminar flow through a SMX mixer 15. Rama Rao et al. ${ }^{16}$ studied experimentally the pressure drop and the distribution of the dispersed phase size also in SMX mixers.

In the summary of the literature review it can be concluded that the RANS approach was usually employed for turbulent flows in static mixers. However, those timeaverage analysis tools of turbulent flows did not accurately reproduce several details of the complex flows. An attempt to improve the simulations was the main motivation for continuing the earlier investigations with the LES model of the L-L flows in the Kenics static mixer.

\section{MODEL DESCRIPTION}

Three different models are commonly used to resolve multiphase flows, i.e. the Volume of Fluid, the Eulerian and the Eulerian-Lagrangian ${ }^{\mathbf{1 7}}$. For the dispersed type of flow the last two types of modelling are used with a separate transport equations for each of the flowing phases.

One of the Eulerian models, included in the commercial Fluent code, is represented by the Mixture model. Each of the two liquid phases is treated as a semi-continuum, interpenetrating each other, which enables transfer of momentum, energy and mass between the phases. The continuity equation (1) for the dispersed phase, which presents the mass balance for that phase, reads

$\frac{\partial}{\partial t}\left(X_{d} \rho_{d}\right)+\nabla \cdot\left(X_{d} \rho_{d} \vec{v}_{m}\right)=-\nabla \cdot\left(X_{d} \rho_{d} \vec{v}_{d r, p}\right)$

where $\rho_{\mathrm{d}}\left[\mathrm{kg} \mathrm{m}^{-3}\right]$ is the density of the dispersed phase (subscript d), $v_{m}\left[\mathrm{~m} \mathrm{~s}^{-1}\right]$ means the velocity of the mixture (subscript $\mathrm{m}$ ) and $v_{d r, p}\left[\mathrm{~m} \mathrm{~s}^{-1}\right]$ is the drift velocity of the dispersed phase. The $v_{d r, d}$ parameter is defined by the algebraic expression (2), where $v_{c d}$ (Eq.3) stands for relative velocities of the continuous (subscript c) and the dispersed phase (d).

$\vec{v}_{d r, p}=\vec{v}_{c d}-\sum_{k=1}^{n} c_{k} \vec{v}_{c i}$

$\vec{v}_{c d}=\vec{v}_{d}-\vec{v}_{c}$

The momentum equation (4) is solved for the mixture and it can be written in the following form

$\frac{\partial}{\partial t}\left(\rho_{m} \vec{v}_{m}\right)+\nabla \cdot\left(\rho_{m} \vec{v}_{m} \vec{v}_{m}\right)=-\nabla p+\nabla \cdot\left[\mu_{m}\left(\nabla \vec{v}_{m}+\nabla \vec{v}_{m}{ }^{T}\right)\right]+\vec{F}_{\text {tot }}$

In equation (4), $\rho_{m}\left[\mathrm{~kg} \mathrm{~m}^{-3}\right]$ stands for the mixture density, $\mu_{m}\left[\mathrm{~kg} \mathrm{~m}^{-1} \mathrm{~s}^{-1}\right]$ is the molecular viscosity of the mixture and $F_{\text {tot }}\left[\mathrm{Pa} \mathrm{m}^{-1}\right]$ means the total additional force per unit area. More details of the Mixture model were described in the previous paper ${ }^{3}$.

The numerical solutions were always obtained for the two approaches, i.e. the Reynolds averaged Navier-Stokes, RANS, and the large eddy simulation, LES. In the RANS method the local instantaneous values for velocity components and the dispersed phase volumetric fraction are replaced by their mean values averaged over the whole turbulence spectrum. On the other hand, in the LES method those instantaneous values are replaced by their counterparts filtered over a part of the spectrum ${ }^{\mathbf{1 8}}$. In those two approaches the effective viscosity replaces the molecular one. However, its main component - turbulent viscosity is differently computed.

The turbulent viscosity, $\mu_{t}\left[\mathrm{~kg} \mathrm{~m}^{-1} \mathrm{~s}^{-1}\right]$, for the RANS method was computed traditionally ${ }^{19}$ from equation (5) and for its evaluation the knowledge of the $k$ and $\varepsilon$ values is essential.

$\mu_{t}=\rho_{m} C_{\mu} \frac{k^{2}}{\varepsilon}$

In equation (5) $k\left[\mathrm{~m}^{2} \mathrm{~s}^{-2}\right]$ denotes the turbulent kinetic energy, $\varepsilon\left[\mathrm{m}^{2} \mathrm{~s}^{-3}\right]$ stands for the turbulence dissipation rate, $\mathrm{rm}$ is the density of the mixture and the model constant $C_{\mu}=0.09$.

A variety of LES models for predicting the sub-grid scale viscosity, $\mu_{S G S}, \quad\left[\mathrm{~kg} \mathrm{~m}^{-1} \mathrm{~s}^{-1}\right]$ can be found in the literature. The first and still the most popular model used for the eddy viscosity evaluation was proposed by Smagorinsky and Lilly ${ }^{9}$. Usually it is expressed as $\mu_{S G S}=\rho\left(C_{s} \Delta\right)^{2}|\widetilde{S}|$

$$
\begin{gathered}
\text { with } \\
|\widetilde{S}|=\sqrt{2 \widetilde{S}_{i j} \widetilde{S}_{i j}}
\end{gathered}
$$

and where $\Delta[\mathrm{m}]$ is the filter width, $C_{s}$ is the Smagornisky constant $\left(C_{s}=0.1\right)$ and $\tilde{S}_{i j}\left[\mathrm{~s}^{-1}\right]$ is the strain rate tensor

based on the filtered velocity field.

The time step of 0.001 [s] applied in the simulation marching procedure was the same as that adopted in the previous work ${ }^{2}$. The total simulation time was assumed at the level of four time scales of turbulence, $4 \tau$, where $\tau$ is defined as $\tau=k / \varepsilon$.

\section{METHODS AND MATERIALS}

Both the RANS and LES numerical simulations were performed for a static mixer equipped with the Kenics inserts (Figure 1 ). The symbols $D[\mathrm{~m}]$ and $h[\mathrm{~m}]$ denote respectively the diameter and length of the Kenics insert. The static mixer consisted of ten inserts twisted by $180^{\circ}$ alternatively: clock-wise and counter clock-wise.

A detailed description of the tube and inserts dimensions was presented elsewere ${ }^{2}$.

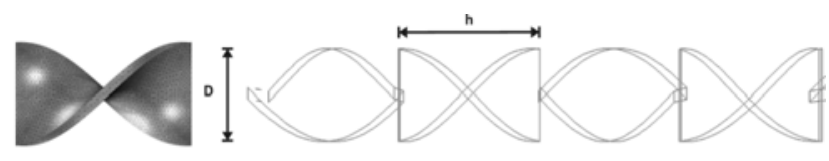

Figure 1. Scheme of the Kenics mixer geometry

The numerical modelling was performed in a similar way to the former work ${ }^{2,3}$ also for three cases (A, B and $\mathrm{C}$ respectively) and using the Fluent 6.2.16 package. Water was used as the continuous phase (index „c", 99\% v/v) whereas silicon oil was used for the dispersed phase (index ,d”, $1 \% \mathrm{v} / \mathrm{v})$. The physical and rheological properties of the two liquids are specified in Table 1, where "Co" denotes the continuous phase and „Dis" the dispersion phase.

The difference in the average density of the mixture, $\bar{\rho}_{a v}$, for Cases $\mathrm{A}$ and $\mathrm{C}$ resulted in a different superficial axial velocity, $\left\langle v_{z}\right\rangle$, of the mixture.

In the steady-state RANS modelling, about 3000 iterations were executed for each of the three cases, which 
Table 1. Properties of the phases

\begin{tabular}{|c|c|c|c|c|c|c|}
\hline Case & \multicolumn{2}{|c|}{$\mathrm{A}, \rho_{\mathrm{c}}>\rho_{\mathrm{d}}$} & \multicolumn{2}{|c|}{$B, \rho_{c}=\rho_{d}$} & \multicolumn{2}{|c|}{ C, $\rho_{c}<\rho_{d}$} \\
\hline Phase & Co & Dis & Co & Dis & Co & Dis \\
\hline Liquids & water & oil & phase "c" & phase "d" & phase "c" & phase "d" \\
\hline$\rho\left[\mathrm{kg} \mathrm{m}^{-3}\right]$ & 998.2 & 900 & 900 & 900 & 900 & 998.2 \\
\hline$\mu[\mathrm{Pa} \mathrm{s}]$ & $1.003 \cdot 10^{-3}$ & $9 \cdot 10^{-4}$ & $1.003 \cdot 10^{-3}$ & $9 \cdot 10^{-4}$ & $1.003 \cdot 10^{-3}$ & $9 \cdot 10^{-4}$ \\
\hline $\bar{\rho}_{a v}\left[\mathrm{~kg} \mathrm{~m}^{-3}\right]$ & \multicolumn{2}{|c|}{997.2} & \multicolumn{2}{|c|}{900} & \multicolumn{2}{|c|}{901} \\
\hline$\left\langle v_{z}>\left[\mathrm{m} \mathrm{s}^{-1}\right]\right.$ & \multicolumn{2}{|c|}{0.414} & \multicolumn{2}{|c|}{0.445} & \multicolumn{2}{|c|}{0.445} \\
\hline$\sigma\left[\mathrm{N} \mathrm{m}^{-1}\right]$ & \multicolumn{4}{|c|}{0.0347} & & \\
\hline
\end{tabular}

allowed reaching the plateau region of the standardized numerical residua.

Numerical integration in time was performed by an implicit scheme. The convection Courant number value of

$C_{k}=\frac{\sqrt{k \delta t}}{h}=\frac{1}{20}$

was accepted and $\delta t[\mathrm{~s}]$ and $h[\mathrm{~m}]$ in Eq. (8) denote the iteration time step and the mean cell width, respectively. In the LES modelling the single time step was $\delta t=10^{-3}[\mathrm{~s}]$ and the total simulation time was $\Delta t=1.0[\mathrm{~s}]$, which resulted from 1000 time steps. Within each time step, 50 SIMPLE iterations were performed to couple velocities and pressure fields.

The converged local and transient values of the velocity components and phase concentrations were recorded for the time steps chosen.

With the adopted spatial and temporal discretization the computing time was of about 40 days to simulate 3 cases.

\section{RESULTS AND DISCUSSION}

In this study the flow field was resolved for $R e=10000$ and for the A, B and C cases using two methods: unsteady LES and steady-state RANS. The local values were recorded for the components of velocity at the point $\mathrm{R}$ in the middle of the fifth insert with coordinates: $x=0.006$ [m], $y=0[\mathrm{~m}], z=0.238[\mathrm{~m}]$. Based on those values, the level of the velocity fluctuation was evaluated for RANS and LES approaches. The current report presents the simulated flow field of the two other velocity components, i.e. the radial and tangential, which allow to estimate the levels of the mean and fluctuating velocity components.

Fig. 2 demonstrates time dependence of the radial velocity component for the A Case at a monitoring point

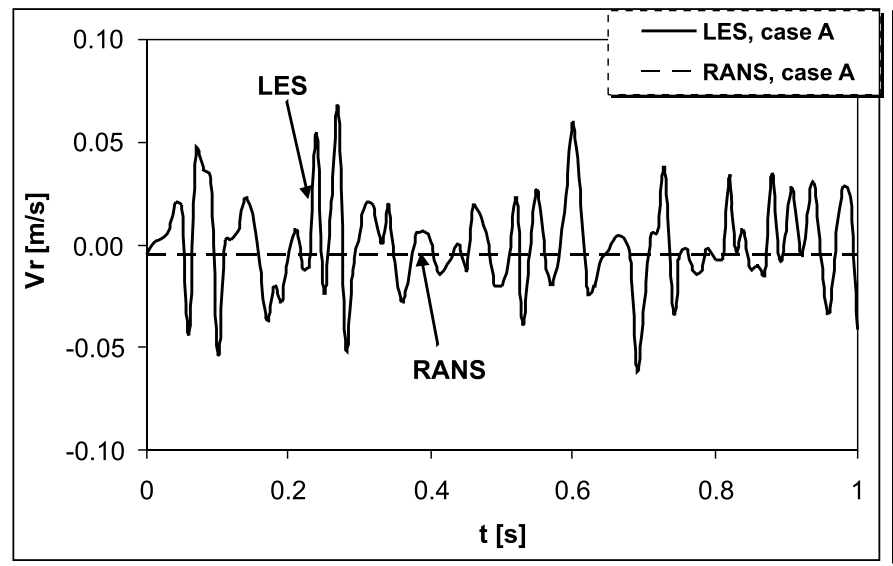

located in the middle cross-section of the fifth Kenics insert. Likewise in the former studies ${ }^{3}$, the results for the time range of $\Delta t=0 \div 1[\mathrm{~s}]$ were analyzed and confirmed the occurrence of the developed turbulence in that direction. Only the two examples with different phase density, i.e. Cases $\mathrm{A}$ and $\mathrm{C}$, are presented here for the sake of brevity since again the simulated B Case did not reveal phase segregation. Similarly to the axial component ${ }^{3}$, fluctuations of the radial velocity component of the two-phase mixture exhibit strong changes in time, see Fig. 2. The local mean value of the radial fluctuating component obtained from LES amounted to 0.024 and $0.026\left[\mathrm{~m} \mathrm{~s}^{-1}\right]$ for the A and C cases, respectively. For the sake of comparison, in the RANS modelling the mean radial velocity at the point was close to $-0.004\left[\mathrm{~m} \mathrm{~s}^{-1}\right]$ and $-0.005\left[\mathrm{~m} \mathrm{~s}^{-1}\right]$ in the Cases $\mathrm{A}$ and $\mathrm{C}$, respectively. Those values are shown with the broken lines in Fig. 2. However, the time averaged radial velocity in the LES modelling was less negative and their estimates were $0.0002\left[\mathrm{~m} \mathrm{~s}^{-1}\right]$ and -0.0018 $\left[\mathrm{m} \mathrm{s}^{-1}\right]$ respectively for the $\mathrm{A}$ and $\mathrm{C}$ Cases.

The standard deviation of the simulated tangential velocity component was significantly higher than that for the radial component and was calculated for the $\mathrm{A}$ and $\mathrm{C}$ Cases respectively as $0.034\left[\mathrm{~m} \mathrm{~s}^{-1}\right]$ and $0.035\left[\mathrm{~m} \mathrm{~s}^{-1}\right]$. Fig. 2 confirms that the LES method is capable of reconstructing the heterogeneity of velocity fluctuations in the turbulent L-L flow in a more realistic manner than in RANS simulations. That conclusion is essential in deciding which simulation method to apply for a given modelling task.

The time history of the fluctuations of the two velocity components can be compared with the help of Fig. 3, which presents graphically the data for the tangential component. In a similar way to the radial velocity, also the tangential exhibits strong instabilities predicted by LES. Nevertheless, their mean values are somewhat lower than those for the steady-state tangential components

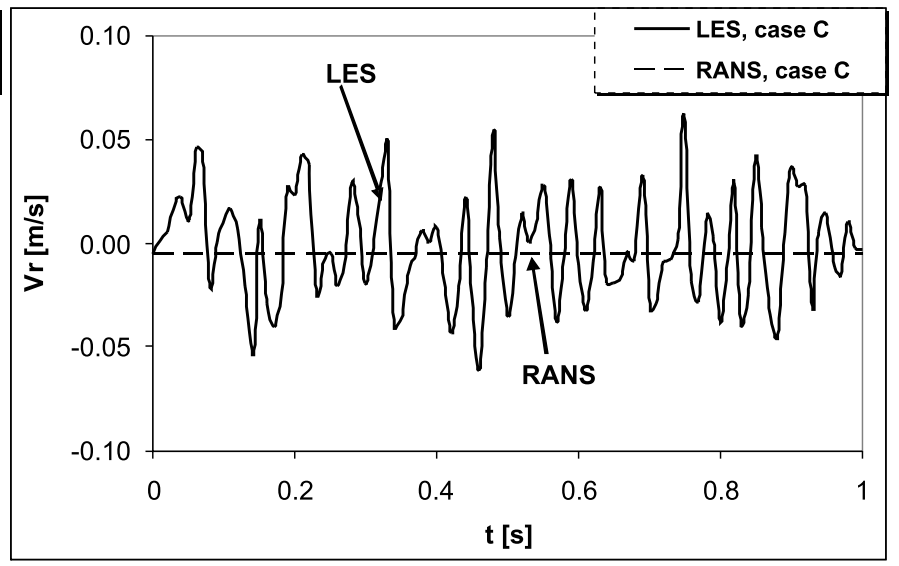

Figure 2. Time dependence of the radial velocity obtained from RANS and LES for Cases A and C, $\operatorname{Re}=10000$ 

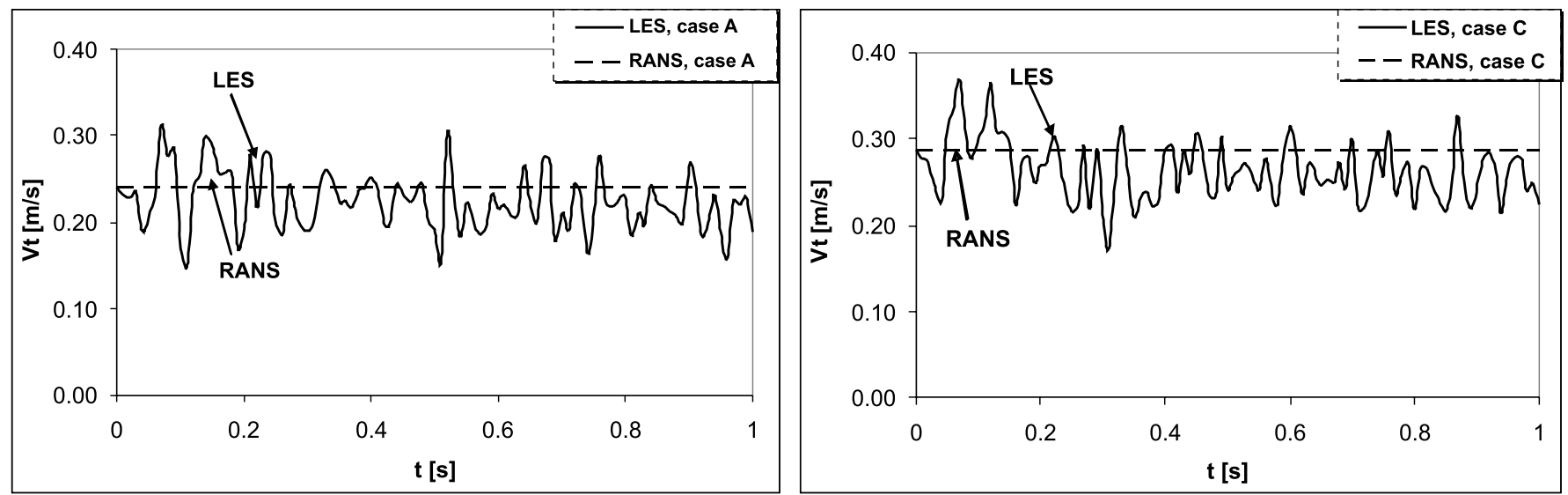

Figure 3. Dependence of the tangential velocity on the time obtained from RANS and LES modelling for case A and C, $\operatorname{Re}=10000$

obtained from RANS. The differences amounted to $6 \%$ and $8 \%$ for the A and C Cases, respectively. Relatively high values of the fluctuating tangential velocity, $v_{t}^{\prime}$, were obtained from the modelling and resulted in $0.034\left[\mathrm{~m} \mathrm{~s}^{-1}\right]$ for the $\mathrm{A}$ and $0.035\left[\mathrm{~m} \mathrm{~s}^{-1}\right]$ for the $\mathrm{C}$ and they had higher simulated magnitude than the radial ones. However, the greatest fluctuations were detected for the axial velocity component ${ }^{3}$, which may be associated with its mean value of $0.41\left[\mathrm{~m} \mathrm{~s}^{-1}\right]$ (case A) and $0.44\left[\mathrm{~m} \mathrm{~s}^{-1}\right]$ (case C) significantly higher than those for the mean radial and tangential values. In the previous report ${ }^{3}$, the standard deviation (mean fluctuation) of the axial component was estimated in LES at the high level of 0.20 and $0.23\left[\mathrm{~m} \mathrm{~s}^{-}\right.$ $\left.{ }^{1}\right]$ respectively for the A and C cases. The combined observations suggest the occurrence of a highly anisotropic turbulence inside Kenics inserts.

In order to help visualize the distributions, contours of the velocity components obtained both from the RANS and LES modelling methods were examined. Typical maps for the tangential component in the mid-cross section of the $5^{\text {th }}$ insert are shown for Cases A and C in Fig. 4.

The analysis of the distributions allows concluding that the velocity contours obtained from the RANS approach are significantly more homogeneous than those obtained in LES. The velocity maps for RANS simulations for Cases A and C do not differ considerably. However, for the LES modelling the maps for Cases $\mathrm{A}$ and $\mathrm{C}$ show significant variations. Closer inspection of such the LES maps revealed strong velocity fluctuations in time and
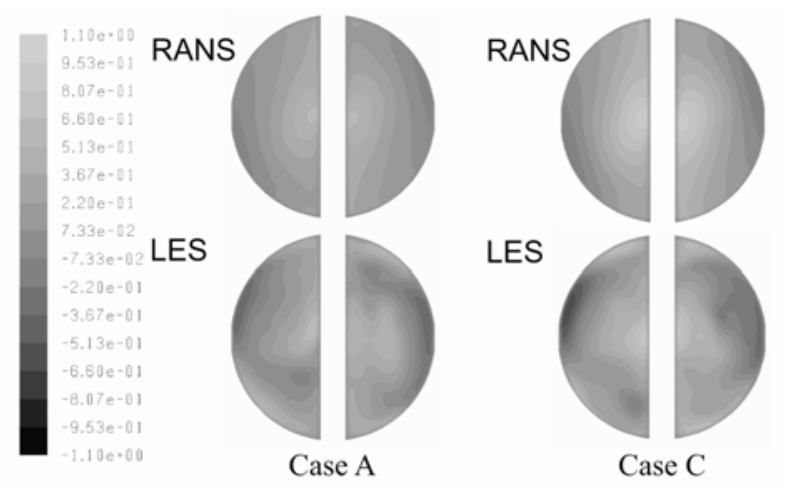

Figure 4. Maps of the tangential velocity in the middle of the fifth insert, Kenics static mixer, $R e=10000$, results from RANS $(\mathrm{t}=0.0[\mathrm{~s}])$ and LES $(\mathrm{t}=1.0$ $[\mathrm{s}])$ lack of symmetry, like in the RANS modelling. Quantitative examples of the fluctuations of two velocity components are shown in Figs. 2 and 3. The highest absolute values of the tangential component were found close to the middle of the inserts and the mixer wall, see Fig. 4. The maps from RANS and LES for the other velocity components (not shown here) and the corresponding Cases also show similar differences between them. However, all of them demonstrate distinct local instabilities of all velocity components reproduced by the large eddy simulation technique, which proves its suitability in modelling turbulent flows.

\section{CONCLUDING REMARKS}

The turbulent flow field generated in the Kenics mixer was numerically simulated by using large eddy simulation (LES) along with the Smagorinsky model for the subgridscales and also by RANS simulation with the $k$ - $\varepsilon$ turbulent model. The computations were run using the general purpose commercial CFD code Fluent. Evolution in the time of the radial and tangential velocity components was analysed in detail.

The results of large eddy modelling a revealed relatively high level of fluctuations of the basic local characteristics of the turbulent flow components, such as the axial, radial and tangential velocity inside the Kenics mixer. The information of this kind cannot be obtained from the RANS modelling where only the average values for the whole turbulence spectrum are directly delivered. Therefore LES gives a much better insight into the local, instantaneous characteristics of the turbulent two-phase flow.

The two velocity components, which are discussed in this paper, along with the axial one analyzed earlier confirm a hypothesis that the turbulence in the Kenics mixer is clearly anisotropic. The three velocities strongly fluctuated in time and space, which is the main feature of turbulent flows. This allows recognizing the LES method as a superior tool for reflecting the structure of the turbulent flow field, especially for the observations of the flow dynamics. That information is of extreme value in predicting the break-up and coalescence effects of the dispersed phase elements in liquid-liquid flows, which depend on the local and instantaneous values of the kinetic energy of turbulence and its dissipation rate. 
In conclusion, large eddy simulations corroborated its universality as a powerful tool for turbulent flow field simulations of liquid-liquid mixtures in static mixers.

\section{LITERATURE CITED}

1. Godfrey, J. Static Mixers in the Process Industries, editors: Harnby N., Edwards M., Nienow A., ButterworthHeinmann, USA, 1992, $225-249$.

2. Jaworski, Z., Murasiewicz, H. \& Pianko-Oprych, P. Large Eddy Simulation of turbulent liquid-liquid flow in Kenics static mixers, Proc. 10th Intern. Conf. Multiphase Flow in Ind. Plant, 2006, Tropea, Italy.

3. Jaworski, Z. \& Murasiewicz, H. CFD of turbulent liquidliquid flow in Kenics static mixers, Proc. 11th Intern. Conf. Multiphase Flow in Ind. Plant, 2008, Palermo, Italy.

4. Byrde, O. \& Sawley, M. Optimization of a Kenics static mixer for non-creeping flow conditions, Chemical Engineering Journal, 1999, 72, 163 - 169 [DOI:10.1016/S13858947(98)00145-4].

5. Szalai, E.S. \& Muzzio, F. Fundamental approach to the design and optimization of static mixers, Aiche J., 2003, 49, 11, 2687 - 2699 [DOI:10.1002/aic.690491103].

6. Song, H. \& Han, S. A general correlation for pressure drop in a Kenics static mixer, Chemical Engineering Science, 2005, 60, 5696 - 5704 [DOI:10.1016/j.ces.2005.04.084].

7. Hobbs, D.M. \& Muzzio, F. Optimization of a static mixer using dynamical system techniques, Chemical Engineering Science, 1998, 53, 18, 3199 - 3213 [DOI:10.1016/S00092509(98)00115-8].

8. Jaworski, Z., Pianko-Oprych, P., Marchisio, D. \& Nienow, A.W. Numerical modelling of turbulent drop breakage in a Kenics static mixer and comparison with experimental data, $12^{\text {th }}$ European Conference on Mixing, 2006, Bologna, Italy.

9. Pope, S. Turbulent Flows, 2000, Cambridge University Press, Cambridge.

10. Hewitt, G.F. \& Vassilicos J.C. Prediction of Turbulent Flows, 2005 Cambridge University Press.

11. Jahoda, M., Mostek, M., Kukukova, A., Machon, V. CFD modelling of liquid homogenization in stirred tanks with one and two impellers using large eddy simulation, Chemical Engineering Research and Design, 2007, 85, A5, $616-625$.

12. Bakker, A. \& Oshinowo, L.M. Modelling of turbulence in stirred vessels using large eddy simulation, Chemical Engineering Research and Design, 2004, A9, 1169 - 1178 [DOI:10.1205/cerd.82.9.1169.44153].

13. Hartmann, H., Derksen, J.J., Montavon, C., Pearson, J., Hamill, I.S. \& van den Akker, H.E.A. Assessment of large eddy and RANS stirred tank simulations by means of LDA, Chemical Engineering Science, 2004, 59, 2419 [DOI:10.1016/ j.ces.2004.01.065].

14. van Wageningen, W., Kandhai, D., Mudde, R. \& van den Akker, H. Dynamic flow in a Kenics static mixer: An assessment of various CFD methods, AIChE Journal, 2004, 50, 1684 - 1696 [DOI:10.1002/aic.10178].

15. Liu, S.J., Hrymak, A.N. \& Wood, P.E. Drop breakup in an SMX static mixer in laminar flow, Canadian Journal of Chemical Engineering, 2005, 83, 793 - 807.

16. Rama Rao, N.V., Baird, M.H.I., Hrymak, A.N. \& Wood, P.E. Dispersion of high-viscosity liquid-liquid systems by flow through SMX static mixer elements, Chemical Engineering Science, 2007, 62, 23, $6885-6896$.

17. Sablani, S.S. et al. Handbook of food and bioprocess modeling techniques, 2007, CRC Press Taylor \& Francis.

18. Wilcox, D. Turbulence Modelling for CFD, 1993, DCW Industries, California.

19. Launder, B. \& Spalding, D. Mathematical Models of Turbulence, 1972, Academic Press Inc. London. 